\title{
Contents, Vol. 24, 1910
}

\section{Inhalts-Verzeichnis}

Original-Arbeiten.

Asmus W., und M ellinghoff , Rud., Klinische und anatomischeBeobachtung eines deletären

Falles von sympathischerOphthalrøie335

Bergmeister, Rudolf, Ein Beitrag zur Gliomfrage. (Hierzu

\section{Taf. XIII-XIV) 320}

Birch-Hirschfeld, A., 7iWX Kenntnis der Tuberkulose der Orbita und des Sehnerven sowie der nach Resektion des Optikus am Bulbus eintretenden Veränderungen. (Hierzu

Tafel VI-VII) 193

Weiterer Beitrag zur Tarsoplastik nach Büdinger (Ver-

wendung von Ohrknorpel zur Lidbildung) $\quad 410$

Orönholm, V., Durch hintere Ethmoidalzellen- und Keilbein-höhlenempyem bedingte ChiasmaAffektion mit Möglich-keit, den Sitz der Läsion innerhalb des Chiasma zu be-stimmen 311

- $\quad$ - Beiträge zur Kenntnis des Enophthalnius traumaticus 479Hesse, Rob., Über Drüsen der Chorioidea und senile Maculaveränderungen. (Hierzu Tafel X) 219

- $\quad$ - Zur Kenntnis der sogenannten präretinalen Blutungen

(Hierzu Tafel XV) 327

Junius, P., Untersuchungen zur Ätiologie des Trachoms.

(Hierzu Tafel XVII-XXI) 383

Köllner, H., Ueber den Wilbrandschen Prismenversuch bei

der Hemianopsie

9

Könnecke, Karl, Beitrag zur Pathologie des Optikuskoloboms.

(Hierzu Tafel XI) 222

Kuhnt, Herm., Ueber einige Verbesserungen der Bindehaut-

Keratoplastik 19

Zur Beseitigung des Entropium organicum am unteren

Lide. (Hierzu Tafel V) 151

Zur operativen Bekämpfung partieller Hornhaut-

staphylome 516

Kuschel, J., Die Verziehungen des Sehnerveneintritts durcli

die Verdehnungen am hinteren Augenpole 488

- IV -

Langenhan, F., Klinische Verwertung der Durchleuchtung desAugenhintergrundes vom Rachen her mit besondererBerücksichtigung der diaphanoskopischen Diagnose intra-okularer Tumoren des Iiinteren Bulbusabschnittes. (Hierzu Tafel I-II) 97

ïnstrumentelle Messung der Zurückdrängbarkeit des

Augapfels in die Augenhöhle 417 
- - Pruning der Transparenz markhaltiger Nerveníasernder N etzhaut und Papille mittels der Durchleuchtung desAugenhintergrundes. (Hierzu Tafel XXII) 512

Lurie, 0. R.: Doppelseitige plastische Iritis bei Erythema

nodosum 458

Mauersberg, P., Hypopyonkeratitis, hervorgerufen durch den

Bacillus pyocyaneus 299

Napp, Otto, Beitrag zur Frage der Entstehung der atypischen

Aderhautkoloborne. (Hierzu Tafel III) 124

Oliveres, A., Ueber einige arthritische Augenbefunde . . . 226

Ollendorf, A., Ueber subkonjunktivale Fibrolysin-Injektionen 30

Penner, C, Ein Fall von seltener Schrotschussverletzung des

Auges ohne Perforation der Bulbushüllen 27

Pichler, Alexius, Das Krankheitsbild des traumatischenEnophthalmus und seine pathologische Anatomie. (Hierzu Tafel XII) 285, 424

Reis, Wilh > ., Ueber diffuse Papillomatose der Cornea. (Hierzu

Tafel IV) 129

Schneider, Wilhelm, Ueber einen Fall von Gliose der Xetzhaut.

(Hierzu Tafel XVI) 332

Terlinck, H., Ein Fall von angeborener Fistel der Tränendrüse 522

Tertsch, Rudolf, Zwei geheilte Fälle von Akustikustunior mit Persistieren der Stauungspapille des einen Falles drei Monate nach der Operation. (Hierzu Tafel VIII-IX) 210

TJwrner, Walther, Ein reflexloser Handaugenspiegel .... 1

Verwey, A., Ueber die Ermüdung beim Lesen in Zusammen-hang mit dem Einfluss der Lage der Blickebene auf denGleichgewichtsstand der Augen 114

Wolfrum, M. G. und Stimmel, K., in Leipzig, Zwei Fälle von

Primäraffekt der Bindehaut. (Hierzu Tafel V) ... 141

Berichte über die deutsehe ophthalmologische Literatur.

Anatomie (II. Semester 1909). Von Prof. Dr. Sobotta in Würz-

burg 34

- V -

Spezielle Patliologie und Therapie. Von Dr. Konrad Schrader

in Gera46, 156, 228

Dioptrik und Anomalien der Refraktion und Akkommodation.

Von Prof. Dr. Max Salzmann in Wien 350

Bericht über die ausländisehe ophthaimologisehe Literatur,

Bericht über die belgische Literatur 1909. Von Dr. H. Terlinck

und Dr. F. Marzorati in Brüssel 524

Bericht über die holländische ophthaimologisehe Literatur des

Jahres 1910. I. Semester. Von Prof. Dr. W. Kosíer-Gzn.

in Leiden und Dr. G. J. Schoute in Amsterdam . . . 342 Bericht über die ungarische Literatur. I. und II. Semester

1910. Von Dr. Jidius Vermes in Budapest 526

Gesellschaftsberiehte.

XXXVI. Zusammenkunft der Ophthalmologischen Gesell-schaft zu Heidelberg am 4., 5. und 6. August 1910. Von

Dr. Juníus in Köln 
82. Versammlung deutscher Naturforscher und Aerzte in

Königsberg i. Pr. Von Dr. Wirths in Königsberg i. Pr. 462Vereinigung der Augenärzte der Provinz Sachsen, Anhalts und

der Thüringer Lande. Von Prof. Braunschweig in Halle 276VI. Versammlung der ungarischen ophthalmologischen Ge-sellschaft in Budapest. Von Doz. Dr. L. v. Blaskovics in Budapest 356

Berliner ophthaimologisehe Gesellschaft

Sitzungen vom 23. Juni und 14. Juli 1910 175

Sitzung vom 27. Oktober 1910

Ophthaimologisehe Gesellschaft in Wien

Sitzung vom 11. Mai 1910

81

„, 22. .Tuni 1910 177

Unfall- und Versicherungskunde. . .84, 180, 278, 367, 470, 534

Therapeutisehe Umsehau $\quad$ 85, 183, 281, 372, 473, 539

Diagnostische Notizen 89, 185, 283, 368, 471.

Aus den Grenzgebieten 544

Literaturverzeichnis 91, 189, 376, 475, 546

Offene Korrespondenz

Tagesnaehriehten

374

Personalien ...96, 192, 284, 382. 478, 548

Berichtigung 192

S. Saehregister $\quad 549$

Namenregister

556 